\title{
Modeling heterogeneities in the crosslinked bacterial sacculus
}

\author{
Garima Rani $\circledast^{1,2}$ and Issan Patri ${ }^{3}$ \\ ${ }^{1}$ The Institute of Mathematical Sciences, C I T Campus, Chennai 600 113, India \\ ${ }^{2}$ Homi Bhabha National Institute, Training School Complex, Anushakti Nagar, Mumbai 400094, India \\ ${ }^{3}$ Chennai Mathematical Institute, SIPCOT IT Park, Siruseri, Chennai, India
}

(Received 21 July 2019; published 28 January 2020)

\begin{abstract}
Examining the design principles of biological materials, in particular, the presence of inhomogeneities in their ultrastructure is the key to understanding the often remarkable mechanical properties possessed by them. In this work, motivated by the question of understanding the effect of variability in the material properties of the peptide crosslinkers on the bulk mechanical properties of the cell wall structure of bacteria, we study a spring system in which variability is encoded by assigning values of spring constants and rupture strengths of the constituent springs from appropriate probability distribution. Using analytical methods and computer simulations, we study the response of the spring system to shear loading and observe how heterogeneities inherent in the system can heighten the resistance to failure. We derive the force extension relation of the system and explore the effect that the disorder in values of spring constant and rupture strength has on load carrying capacity of the system and failure displacement. We also study a discrete step shear loading of the system, exhibiting a transition from quasibrittle to brittle response controlled by the step size, providing a possible framework to experimentally quantify the disorder in analogous structures. The model studied here will also be useful in general to understand fiber bundles exhibiting disorder in the elasticity and rupture strengths of constituent fibers.
\end{abstract}

DOI: 10.1103/PhysRevResearch.2.013090

\section{INTRODUCTION}

Biological structures are some of the most sophisticatedly engineered materials, whose design principles continue to lend ideas for solving common place as well as esoteric engineering riddles [1-3]. A pervasive feature of the design of several biological materials is the presence of disorder in their ultrastructural components [4,5]. However, this cannot be termed as mere accident in light of the often pivotal role played by the disordered fine structure of several key biological materials. Numerous interesting examples of this phenomena can be given, including the role of nonidentical molecular motors in actomyosin contractility [6] and the optimized hierarchical structure with highly irregular setup of bone resulting in remarkable orders of toughness and stiffness $[4,7]$.

One of the most fascinating naturally occurring biomolecule is the peptidoglycan (PG) mesh, the primary component of the cell wall of bacteria. The PG mesh, in rod shaped Gram negative bacteria like Escherichia coli, consists of stiff glycan chains arranged roughly in the circumferential direction, crosslinked intermittently by peptide bonds $[8,9]$ (see Fig. 1). It is a testament to the versatility of this structure that it is able to satisfy a wide array of necessary mechanical requirements, including being stiff enough to bear the

Published by the American Physical Society under the terms of the Creative Commons Attribution 4.0 International license. Further distribution of this work must maintain attribution to the author(s) and the published article's title, journal citation, and DOI. internal turgor pressure but being elastic enough to allow for elongation, apart from being sufficiently resistant to failure due to crack propagation [8-10].

Like many biological materials, the PG mesh also exhibits disorder that is evident in its ultrastructure-this includes the length distribution and orientation of glycan strands and location and degree of crosslinking across the cell wall [11-15]. Nonetheless, the relevance of these features of disorder on the mechanical properties of the cell wall has not been well studied, even as it can shed light on a number of experimental observations of the cell wall. For instance, in a previous work [16], we had explored the mechanical effect of length distribution of the glycan strands and had shown how terminally crosslinked smaller length glycan strands can enhance the toughness of the cell wall, giving an explanation of experimental observations of the presence of shorter length glycan strands and the preference for crosslinking to happen at the ends of the glycan strands [11,14].

The cell wall is continually remodelled in the cell, with cleaving of older crosslinks under the action of hydrolases, incorporation of new cell wall material into it and the consequent formation of crosslinks $[8,10,17]$. This results in the presence of newer crosslinks as well as hydrolase-degraded older crosslinks in the cell wall. While the exact mechanical effect of hydrolases on the peptide crosslinkers is unclear, this effect can result in the lowering of rupture strength of the bond or lowering of the stiffness of the bond or both. The presence of heterogeneities in the mechanical properties of the crosslinkers is further evidenced by experimental observations in Ref. [12], where isolated cell wall fragments subjected to sonication showed an immediate drop in the degree of crosslinking that persisted even as the structure remained 


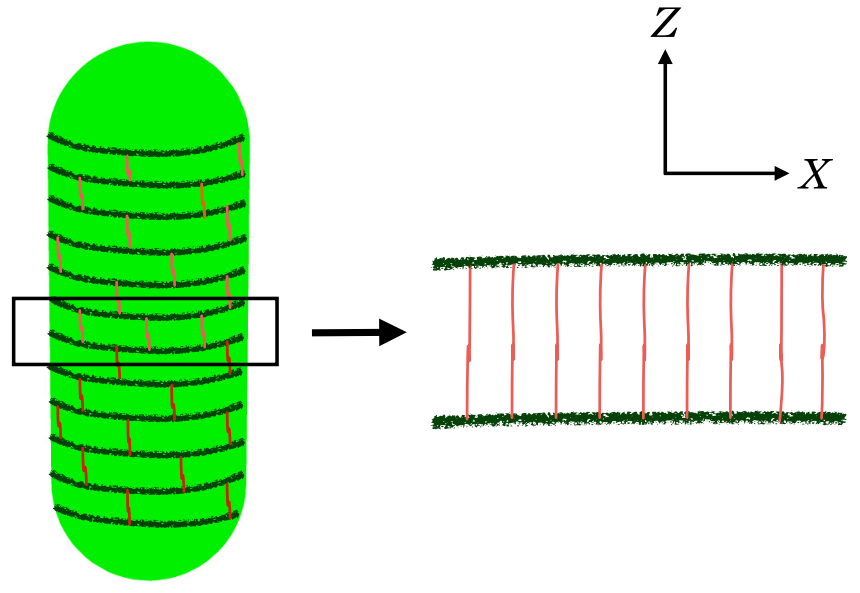

FIG. 1. The peptidoglycan (PG) mesh in E.coli cell wall has stiff glycan strands aligned roughly in the circumferential direction, crosslinked by short peptide bonds. This mesh can be visualized as an ensemble of stiff interfaces and linear springs.

intact, before becoming relatively constant after a period of time. This suggests that the crosslinkers in the cell wall act in a heterogeneous manner under loading, due to differences in their elasticity and rupture strengths. A very natural question then is to understand effect of the variability in the mechanical properties of the crosslinkers, specifically their strength and elasticity, on the bulk mechanical response of the cell wall. Motivated by this and as a first step, here we explore a spring system akin to a Fiber bundle model (FBM) [18,19], in which we incorporate variability in both the spring constant of the constituent springs as well as their rupture strengths and study the response of the system to shear loading. We first consider the case when the distribution of the values of the spring constants and rupture strengths are independent. Using analytical methods and computer simulations, we derive the force extension relation and show that in general wider variability in the elasticity of springs can ensure more robust resistance to failure but it also lowers the load capacity whereas lower variability results in a more brittle response to loading even as the load capacity increases. We also deduce that the value of the displacement at failure does not depend on the lower limit of the distribution of the rupture strengths of the springs, though the load capacity of the system increases as the variability in rupture strengths of constituent springs decreases. This suggests that a possible mechanism for hydrolytic action to act on the crosslinkers in a safe manner while ensuring sufficient load bearing ability is by maintaining high order of variability in the spring constants while limiting the variability in the rupture strengths.

Next, we examine a step wise loading regime which allows us to exhibit a quasibrittle to brittle transition as the load per step increases. This transition, which is a feature that seems universal in systems which have inhomogeneities built into their ultrastructure, has been observed in other natural materials, e.g., snow [20], and can be useful tool to experimentally detect such inhomogeneities present in the system. Finally, we also study the case when the distributions of the spring constants and the rupture strengths of the constituent springs in the system are (positively or negatively) correlated. We show that while in the case of positive correlation, the response to loading is considerably brittle, negative correlation of spring constant and rupture strength values results in quasibrittle behavior mimicking the independent case, although the maximum load carrying capacity drops as compared to independent case. This suggests the likelihood of the spring constant and rupture strength having independent distributions to ensure optimal load carrying capacity as well as resistance to failure in natural systems, including the crosslinkers in the peptidoglycan mesh.

\section{MODEL}

Drawing inspiration from the crosslinked mesh like structure of peptidoglycan in the bacterial cell wall, we study a system of springs placed in the $X-Z$ plane, consisting of two rigid surfaces, aligned in the $X$ direction and linear springs connecting them, aligned in the $Z$ direction. The rigid surfaces correspond to two adjacent circumferential crosssections of the peptidoglycan mesh, consisting of axially adjacent, long length glycan strands, while the springs correspond to the crosslinkers joining the two glycan strands (see Fig. 1). The number of springs connecting the upper and lower surface, denoted $N$, is determined by the degree of crosslinking present in the cell wall: in E.coli, observed degree of crosslinking $\sim 30 \%-50 \%[10,14]$ and with radius of a typical cell $\sim 500 \mathrm{~nm}$ [21], we estimate roughly 1000 peptides stems crosslinked between two adjacent circumferential crosssections of the cell. Therefore, we take $N=1000$ in all simulations in this work. Further, since glycan strands are an order of magnitude more stiff than the peptide bonds [22], for simplicity we assume that upper and lower surfaces are rigid with no local deformations caused by exerted forces. The springs in the system are taken to be linear, with finite rupture strength, which limits the force that a spring can endure before rupturing. We encode variability in the system by taking values of the spring constants and the rupture strengths of the springs from an appropriate joint probability distribution over the region $\left[k_{1}, k_{2}\right] \times\left[f_{1}, f_{2}\right]$ with the upper limits $f_{2}$ and $k_{2}$, which for illustrative purposes, we fix to be equal to 1 . Our aim is to tune the range of variability by altering the values of $f_{1}$ and $k_{1}$ and to see the possible effect on the response of system to loading. In our model, the newly formed crosslinks correspond to springs which are stiffer and have higher rupture strengths while older crosslinks under effect of hydrolases and mechanical stress, are taken to be relatively weaker and less stiff. Therefore, to tune the range of variability in our model, we vary the values of $f_{1}$ and $k_{1}$ while keeping $f_{2}$ and $k_{2}$ fixed. We note that our theoretical setup is independent of the values of the upper limit and our results will not change qualitatively when a change is effected in the values of the upper limit.

In this work, we study shear loading of the spring system. As mentioned before, purified cell wall fragments of E.coli have been subjected to sonication [12], a method of cell disruption acting by shear deformation [23] (in general, shearing is a standard and successful method for performing cell disruption experiments [24]). In our case, the upper surface is displaced by application of force while the lower surface is kept fixed (see Fig. 2). For simplicity, we assume that adjacent springs maintain the position of their link with the 


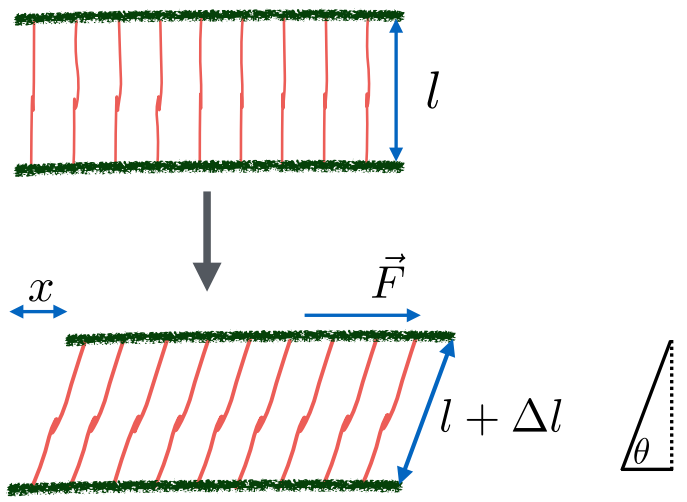

FIG. 2. Schematic representation of spring system considered. Two adjacent surfaces at distance $l$ from each other joined by $N$ linear springs subjected to shear displacement $x$. This results in an extension $\Delta l$ in each spring, with each spring at an angle $\theta$ to the lower surface, given by $\tan \theta=l / x$.

upper and lower surfaces, upto their rupture, ensuring that no sliding motion of the springs occurs. In this case, the elongation is the same for every spring, since the displacement of the point of contact of each spring on the upper surface due to the application of the force, will be the same (equal to displacement of the upper surface). We also take all springs in the system to have the same rest length, equal to the distance between the upper and lower surface (corresponding to the interglycan spacing and denoted as $l$, see Fig. 2). In general, though, it is possible that the peptide crosslinkers in the peptidoglycan sacculus have variable rest lengths, which can potentially induce interesting phenomena, for instance emergence of residual stress in the system due to mismatch of the rest lengths of the crosslinkers with interglycan spacing. This, and yet other modes of heterogeneity in the peptidoglycan sacculus, will be addressed in a subsequent work.

\section{A. Single spring under shear load}

We first write down the equations for a single spring with spring constant $k$ and rupture strength $f$, under similar shear loading as described above. In general, the system can be loaded in two ways-force controlled loading and displacement controlled loading.

For force controlled loading, we assume that a force $F$ is exerted on the upper surface, causing a displacement $x$ of the upper surface and resulting in a stretching of the spring by $\Delta l$. The force in the direction of spring elongation is then given by $F_{\theta}=k \Delta l$. Noting that $\sin \theta=\frac{l}{l+\Delta l}$ and using force balance, we get

$$
\frac{F}{k l}=\left(\frac{1}{\sin \theta}-1\right) \cos \theta,
$$

which gives us a polynomial equation for $\sin \theta$ as

$$
R^{2} \sin ^{2} \theta=(1-\sin \theta)^{2}\left(1-\sin ^{2} \theta\right)
$$

with $R=F /(k l)$. In our case, since $0<\theta<\frac{\pi}{2}$, we have $0<$ $\sin \theta<1$ and it is then easy to see that Eq. (2) has a unique solution. So, solving for $\sin \theta$ using Eq. (2), we can calculate the displacement $x$ in the $X$ direction as

$$
x=\sqrt{\left(\frac{l}{\sin \theta}\right)^{2}-l^{2}}
$$

and the shear strain in then given by $\epsilon=x / l$. In case of displacement controlled loading, a displacement $x$ is given to the upper surface keeping the lower surface intact. The extension $\Delta l$ of the spring is given by

$$
\Delta l=\sqrt{l^{2}+x^{2}}-l .
$$

The force $F$ inducing the extension $x$ on the upper surface is given as

$$
F=F_{\theta} \cos \theta=k \Delta l \frac{x}{l+\Delta l} .
$$

In this work, we carry out a study of displacement controlled loading of the spring system since typically displacement can be measured in an easier and more precise manner.

\section{B. Constitutive behavior of spring system}

We now consider the case of the spring system, undergoing displacement controlled shear loading (see Fig. 2), having $N$ springs, with spring constants $k_{i}$, for $i=1,2, \ldots, N$, where $\mathrm{N}$ is the total number of peptides between two interfaces. The effective spring constant of the $\mathrm{N}$ parallel springs is

$$
k=k_{\text {eff }}=\sum_{i=1}^{N} k_{i} .
$$

The rupture strength of the springs is denoted $f_{i}, i=$ $1,2, \ldots, N$. Note that since the shear strain is the same for all (intact) springs, we can calculate the longitudinal elongation of each spring $\Delta l$ as in Eq. (4) and thus the force on the $i$ th spring is

$$
\sigma_{i}=k_{i} \Delta l .
$$

For all springs $i$ for which $\sigma_{i}$ exceeds the rupture strength $f_{i}$, the spring breaks. The force being exerted in the $X$ direction on the system is then given by

$$
F=\sum_{\text {intact springs }} \frac{\sigma_{i} x}{\sqrt{l^{2}+x^{2}}},
$$

which describes the constitutive behavior of the bundle.

Consider now the special case where the springs all have same spring constant $k$, the force on all springs is the same, $\sigma=k \Delta l$, when a displacement $x$ is given to the upper surface with $\Delta l$ as in Eq. (4). Further assuming that all the springs have the same rupture strength $f$, the force on the system is given by $F_{x}=N \sigma x / \sqrt{l^{2}+x^{2}}$, when $\sigma<f$ and the system collapses when $\sigma$ exceeds $f$. However, as mentioned above, it has been observed that cell wall fragments of E.coli when subjected to sonication, show an immediate drop in the degree of crosslinking, during which the cell wall structure remains intact [12]. This is however at odds with the behavior that a system of springs with all springs having same spring constant and rupture strengths exhibits under shear deformation, so we rule out this case and assume that the values of the spring constants and the rupture strengths displays some measure of variability. 


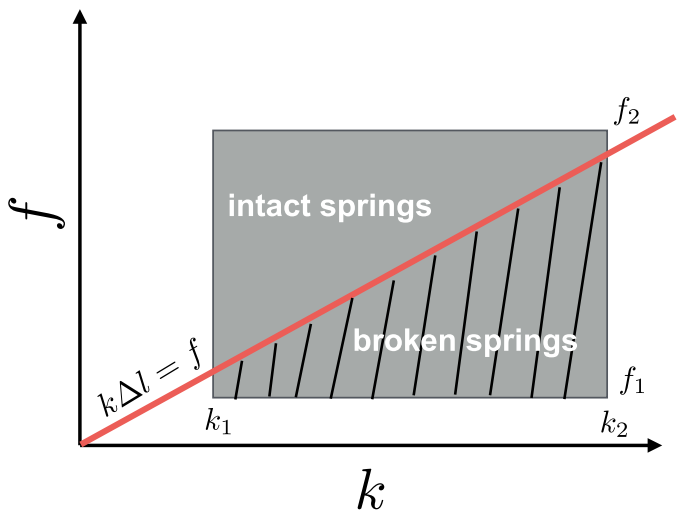

FIG. 3. The joint probability distribution of the values of spring constants and rupture strengths of the constituent springs is distributed on $\left[k_{1}, k_{2}\right] \times\left[f_{1}, f_{2}\right]$. When a shear displacement $x$ is applied to the system, resulting in spring extension $\Delta l$, the fraction of broken springs are those whose spring constant and rupture strength lie in the shaded region, below the line $k \Delta l=f$.

To probe the effect of statistical variability in the elasticity and rupture strengths of the springs, we assume that the springs in the system are assigned the values of their respective spring constants and rupture strengths from a joint probability distribution, denoted $p$. So, in particular, the fraction of springs with spring constant in the interval $[a, b]$ and threshold values in the interval $[c, d]$ is

$$
\int_{a}^{b} \int_{c}^{d} p(k, f) d k d f
$$

Suppose now that a displacement $x$ is imposed on the upper surface keeping the lower surface fixed. The extension in any spring is given by $\Delta l$, as given in Eq. (4). Since only those springs will survive whose spring constant $k$ and rupture strength $f$ satisfy $k \Delta l<f$ (see Fig. 3), in other words, springs whose rupture strength is more than the current load, so the fraction of surviving springs at given extension $x$ is

$$
\frac{N_{s}}{N}=\iint_{\{(k, f): k \Delta l<f\}} p(k, f) d k d f,
$$

and we have

$$
F_{\theta}=N \Delta l \iint_{\{(k, f): k \Delta l<f\}} k p(k, f) d k d f,
$$

and then the force extension relation is given by $F=$ $F_{\theta} \cos \theta=F_{\theta} x /(l+\Delta l)$ as in Eq. (5). We note here that in our spring model, the springs, which represent peptide crosslinkers, have been taken to be linear. However, one can study models in which the crosslinkers follow nonlinear force extension relations by suitably modifying our theoretical framework, though this complication is not studied here.

\section{Simulation details}

We carry out computer simulations to compare with and confirm the theoretical framework laid out in the previous section, for understanding the effect of variability in the material properties of the springs. Specifically, for a given spring system, consisting of $N$ springs denoted by $i=1,2, \ldots, N$, the $i$ th spring is assigned a tuple $\left(k_{i}, f_{i}\right)$, where $k_{i}$ is its spring constant and $f_{i}$ denotes its rupture strength. To study the effect of variability on the mechanical properties of the system, values $\left(k_{i}, f_{i}\right)$ are drawn randomly from appropriate joint probability distribution for every $i$. Given shear displacement $x$, spring extension $\Delta l$ is calculated using Eq. (4) and the force $\sigma_{i}$ on the $i$ th spring is then calculated using Eq. (7). If $\sigma_{i}>f_{i}$, then the spring is considered ruptured and its spring constant $k_{i}$ is assigned value 0 . The force extension relation is then calculated using Eq. (8). The force extension relation and other simulations in the text are obtained by averaging over 100 realizations in each case.

\section{RESULTS}

We now study the effect that the variability in the spring constants and rupture strengths has on the constitutive behavior of the bundle. We first consider the case where the distributions of the spring constants and rupture strengths of the springs in the system are taken to be independent.

\section{A. Effect of variability on constitutive behavior}

Since the distributions of the spring constants and rupture strengths are taken to be independent, so the joint probability distribution $p(k, f)$ [see Eq. (9)] will decompose as product of the marginal distributions $p_{1}$ and $p_{2}$, giving $p(k, f)=$ $p_{1}(k) p_{2}(f)$. We analyze the case where the values of the spring constant and the rupture strength come from uniform distributions. So, with values of the spring constant and the rupture strength lying in $\left[k_{1}, k_{2}\right]$ and $\left[f_{1}, f_{2}\right]$, respectively, we get that

$$
p(k, f)=p_{1}(k) p_{2}(f)=\frac{1}{\left(k_{2}-k_{1}\right)\left(f_{2}-f_{1}\right)} .
$$

To compute the force extension relation, we note that in this case, the integral in Eq. (11) can be evaluated as follows: we define $\eta=\min \left(\max \left(k_{1} \Delta l, f_{1}\right), f_{2}\right)$. Then we have

$$
\begin{aligned}
& \iint_{\{(k, f): k \Delta l \leqslant f\}} k p(k, f) d k d f \\
& \quad=\int_{\eta}^{f_{2}} d f\left(\int_{k_{1}}^{\min \left(k_{2}, f / \Delta l\right)} k p(k, f) d k\right)
\end{aligned}
$$

which gives

$$
F_{\theta}=N \Delta l \int_{\eta}^{f_{2}} d f\left(\int_{k_{1}}^{\min \left(k_{2}, f / \Delta l\right)} k p(k, f) d k\right)
$$

and the force extension relation is then

$$
F=N \frac{x \Delta l}{l+\Delta l} \int_{\eta}^{f_{2}} d f\left(\int_{k_{1}}^{\min \left(k_{2}, f / \Delta l\right)} k p(k, f) d k\right)
$$

with $\Delta l$ given as in Eq. (4) as a function of the shear displacement $x$.

In Fig. 4, using Eq. (15) and computer simulations, we compute the force extension curve and the surviving fraction of springs as a function of the shear extension $x$, acting on a fully intact bundle, with the values of spring constants and rupture strengths of the springs in the system drawn from 

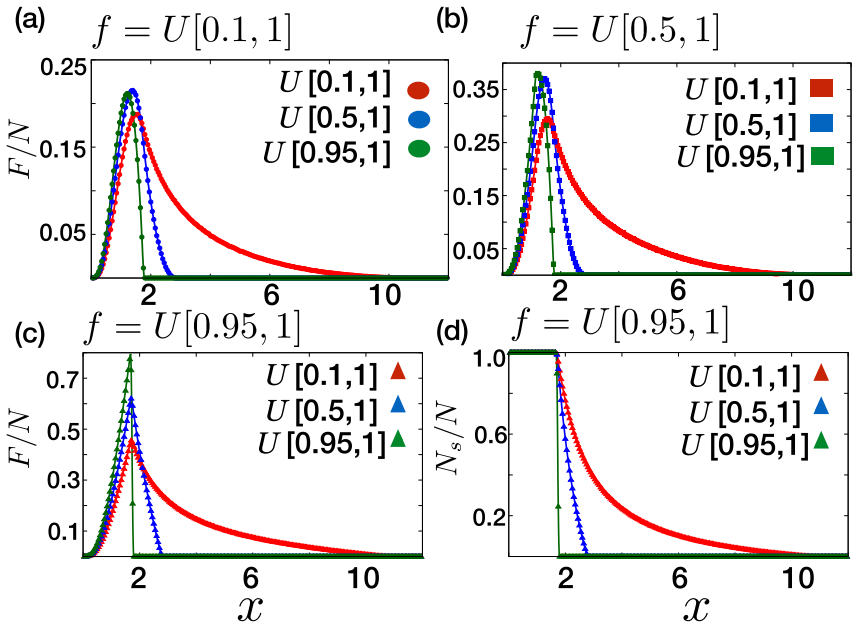

FIG. 4. Force-extension curve with distribution of $f$ taken: (a) $f=U[0.1,1]$, (b) $U[0.5,1]$, and (c) $U[0.95,1]$. In each case, three plots are drawn with probability distribution of $k$ taken as $U[0.1,1], U[0.5,1]$, and $U[0.95,1]$. (d) Fraction of surviving springs is drawn as a function of displacement for $f=U[0.95,1]$. We compare the curve derived analytically from Eqs. (10) and (15) (solid lines) and simulations (solid points) for all the cases. We have taken $N=1000$ and $l=1$. In all the graphs, we note that the system with $k$ distribution $U[0.95,1]$ collapses first while system with $k$ distribution $U[0.1,1]$ collapses last.

uniform distributions over different intervals. In all cases, simulations show excellent agreement with the theoretical computations. We observe a very interesting contrast: in each case, the maximum load that the bundle takes, given by the maximum of the force-extension curve, is highest in the case when springs in the bundle are the stiffest, given in Fig. 4 when the spring constants are uniformly distributed on $[0.95,1]$. However, the extension at which the bundle fails, is maximized in the case when the values of the spring constants is distributed over a wider range, which in Fig. 4 is given by $[0.1,1]$, while bundle with stiffest springs show a brittle response to the shear loading. This suggests that while the load bearing ability of the system is enhanced by the presence of stiff springs, the toughness of the system or the resistance of system to mechanical failure is enhanced by the presence of heterogeneities in the system. This also highlights the standard engineering problem of fabricating materials with high degrees of load bearing ability and toughness, something that nature seems to excel in, with biomaterials like bone and nacre exemplifying this property $[4,25]$. We also note that the maximum load increases as the variability of the distribution of rupture strength decreases [Fig. 4(c)]. Thus an ideal scenario to ensure high load bearing ability and resistance to mechanical failure is to increase the variation in the value of spring constants while keeping the rupture strength high with little variation. This suggests that a possible mechanism for hydrolytic action on the crosslinkers could result in ensuring wider variability in the elasticity of the crosslinkers while showing little effect on their rupture strengths, so as to secure the viability of the structure which has to bear load even as it is being remodelled continually.
We now estimate the shear displacement $x_{i}$ at which spring breakage is initiated and $x_{f}$ at which the system fails. We observe from Fig. 4(d) that $x_{i}$ remains unchanged even as the variability in the values of spring constants is changed. This suggests that $x_{i}$ is independent of $k_{1}$. Similarly, we also observe from Figs. 4(a)-4(c) that $x_{f}$ stays the same in all three cases once the distribution of spring constant is fixed, which suggests that it is independent of $f_{1}$. To understand this interesting phenomena, we estimate $x_{i}$ and $x_{f}$ as follows: note that the ratio of rupture strength and the spring constant $f / k$ for springs in the system takes value in the range $\left[f_{1} / k_{2}, f_{2} / k_{1}\right]$, since the joint distribution $p$ is supported on $\left[k_{1}, k_{2}\right] \times\left[f_{1}, f_{2}\right]$. Therefore breaking of springs is initiated when the displacement ensures that the spring elongation $\Delta l \approx f_{1} / k_{2}$ and system failure occurs when $\Delta l \approx f_{2} / k_{1}$. We can then estimate the displacement $x_{i}$ at which spring failure is initiated and the displacement $x_{f}$ at which the system collapses using Eq. (4), which gives

$$
x_{i} \approx \sqrt{\left(l+\left(f_{1} / k_{2}\right)\right)^{2}-l^{2}}=\frac{\sqrt{\left(2 l k_{2}+f_{1}\right) f_{1}}}{k_{2}}
$$

and similarly we have that the bundle fully breaks when $\Delta l=$ $f_{2} / k_{1}$. So, then we have

$$
x_{f} \approx \sqrt{\left(l+f_{2} / k_{1}\right)^{2}-l^{2}}=\frac{\sqrt{\left(2 l k_{1}+f_{2}\right) f_{2}}}{k_{1}} .
$$

It follows from Eq. (17) that $x_{f}$ depends on the weaker springs that have very high rupture strength, with the extreme case given by springs having spring constant $k_{1}$ and rupture strength $f_{2}$. It is independent of the values $f_{1}$ and $k_{2}$, which represent the values for the stiffest springs which have the lowest rupture strength. These values however determine $x_{i}$ [see Eq. (16)]. Since the displacement is the same for all springs, springs with high stiffness and low rupture strength are the first to rupture. To check this, we perform simulations of bundle of 1000 springs with values of spring constant derived from the distribution $U\left[k_{1}, 1\right]$, for varying $k_{1}$, while the rupture strength of the springs is derived from fixed uniform distribution, to compute the value of $x_{i}$ [plotted in Fig. 5(b) as a function of $k_{1}$ ] and $x_{f}$ [plotted in Fig. 5(d) as a function of $k_{1}$ ]. We also perform simulations keeping the distribution of spring constants to be fixed and the rupture strength distribution to be $U\left[f_{1}, 1\right]$ with varying $f_{1}$ to compute the values of $x_{i}$ [plotted in Fig. 5(a) as a function of $f_{1}$ ] and $x_{f}$ [plotted in Fig. 5(c) as a function of $f_{1}$ ]. In all cases, the simulations results agrees well with the analytical results. We observe from Fig. 5(b) that the value of $x_{i}$ is approximately constant in all three cases as $k_{1}$ varies, in good agreement with Eq. (16), while increasing with value of $f_{1}$ approximately linearly. On the other hand, the value of $x_{f}$ remains constant in all three cases as $f_{1}$ varies, as in Fig. 5(c), while showing a power law dependence on $k_{1}$. This, in particular, reiterates that the ideal scenario to ensure high value of both $x_{f}$ and load capacity arises when $k_{1}$ has a low value while the value of $f_{1}$ is high. This is because a low value of $k_{1}$ ensures that $x_{f}$ has a high value. But the value of $x_{f}$ remains relatively constant as $f_{1}$ changes. Therefore a high value of $f_{1}$, while keeping $x_{f}$ unchanged, limits the variability and hence, increases the load capacity. To understand this effect, we can make a rough estimation of the shear displacement at failure in case of the 
(a)

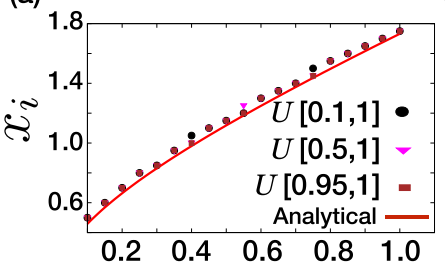

(c)

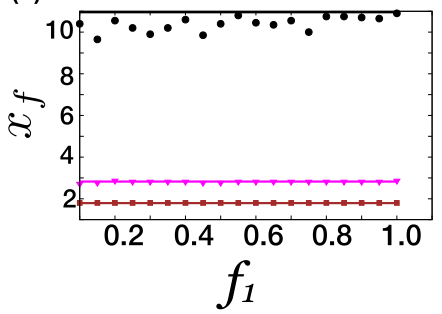

(b)

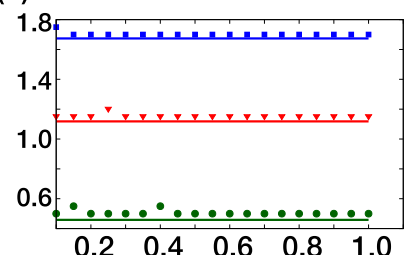

(d)

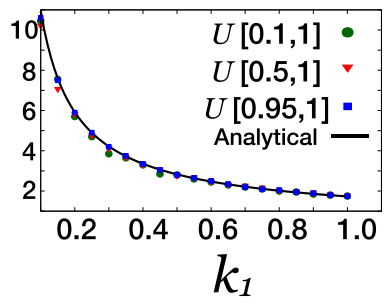

FIG. 5. [(a) and (b)] Displacement at which spring rupturing is initiated, $x_{i}$, is plotted as a function of $f_{1}$, for different distributions of $k_{1}$ as labeled and as a function of $k_{1}$, for different distributions of $f_{1}$, respectively, using Eq. (16) (solid line) and simulations (points). [(c) and (d)] Displacement at which system fails, $x_{f}$, is plotted as a function of $f_{1}$ for different distributions of $k_{1}$ and as a function of $k_{1}$, for different distributions of $f_{1}$ as labeled, respectively, using Eq. (17) (solid line) and simulations (points).

bacterial cell wall as the elasticity of the crosslinkers varywith the spring constant of peptide crosslink estimated as $k_{2} \sim 10^{-2} \mathrm{~N} / \mathrm{m}$ [26] and estimating the rupture strength of the crosslink as $f_{2}=\sqrt{2 E k_{2}}$ where $E \sim 300 \mathrm{~kJ} / \mathrm{mol}$ is the dissociation energy of the covalent crosslinking bond, we get $x_{f}<10 \mathrm{~nm}$ when there is very little variation with $k_{1} \approx k_{2}$ while $x_{f} \sim 10^{2} \mathrm{~nm}$ when there is variation with $k_{1} / k_{2} \approx 0.1$. This calculation, though only a very rough estimation, shows how effectively variation in the elasticity of the crosslinkers can offer high degree of protection from mechanical failure.

Another interesting parameter of the system is the displacement at which the force extension curve attains its maxima, denoted $x_{m}$. As explained in Appendix, it is always true that $x_{i} \leqslant x_{m} \leqslant x_{f}$, it is interesting to observe from Eqs. (16) and (17) that as the variability in $k$ and $f$ is limited with $f_{1} \approx f_{2}$ and $k_{1} \approx k_{2}$, we have that $x_{i} \approx x_{m} \approx x_{f}$ in this case, which highlights the decidedly brittle response to loading of the system in this case, with the spring breaking being initiated, the system attaining its load maximum and system failure all occurring when virtually the same shear displacement has been applied to it. As the parameters $f_{1}$ and $k_{1}$ change, the displacement $x_{m}$ shows rather intriguing crossover behavior: when $f_{1} / k_{1} \ll 1, x_{m}$ exhibits independence from the value of $f_{1}$ but as $f_{1}$ approaches $k_{1}$ and becomes greater than it, this behavior crossover to one where there is a dependence of $x_{m}$ on $f_{1}$. This behavior is explored in detail in Appendix.

\section{B. Loading regimes}

We now impose specific loading regimes on the spring system and study its response. Specifically, given displacement $x(t)$ imposed on the upper surface as a function of time $t$, we compute the fraction of surviving springs as a function of time, which we denote $u(t)=N_{s}(t) / N$. We explore two

(a)

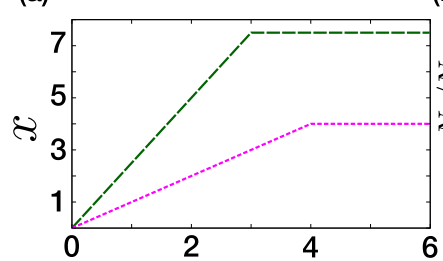

(c)

(b)

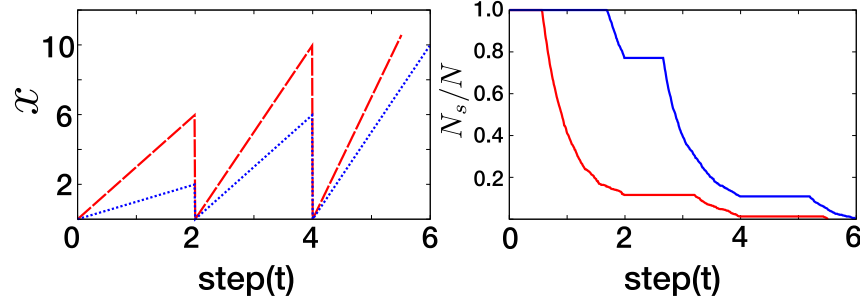

FIG. 6. [(a) and (c)] Loading regimes $x(t)$ are plotted, given shear displacement at time $t$ and the corresponding response of the system to these loading regimes in form of the fraction of surviving springs is plotted as a function of time in (b) and (d), respectively. We note that in each case, for loading regime with higher loads, spring breakage, that is $N_{s} / N<1$, is initiated faster.

loading regimes: (a) where $x(t)$ increases linearly with time before becoming constant and (b) where $x(t)$ increases linearly and then drops to 0 and this cycle is continued, ensuring each time that the load peaks at a level higher than the previous cycle [see Figs. 6(a) and 6(c)]. We note that the response in the first case is an almost immediate drop in the fraction of surviving springs $u(t)$, which then stabilizes when the loading itself stabilizes. We note the similarity in the response to this loading regime to experimental observations in Ref. [12], where the degree of crosslinking of isolated E.coli sacculi subjected to sonication showed an immediate decrease before becoming constant. In the second case, the response to the load results in a calibrated fall in the fraction of surviving springs. When $x(t)$ is increasing, $u(t)$ registers a fall but when $x(t)$ drops to 0 and subsequently increases, $u(t)$ remains constant till $x(t)$ goes beyond the previous peak whence $u(t)$ starts to drop again and this cycle continues.

We now consider another loading regime of the spring system, by stepwise increase of the shear displacement. In other words, loading is provided in discrete steps of shear displacement, $\Delta x$ in each step. We estimate the number of steps to failure in this case. As before, the spring constant and the rupture strength derive values from joint distribution $p(k, f)$ supported fully on $\left[k_{1}, k_{2}\right] \times\left[f_{1}, f_{2}\right]$. The number of steps to failure, denoted $n_{f}(x)$ is given by

$$
n_{f}(\Delta x)=n_{1}(\Delta x)+n_{b}(\Delta x),
$$

where $n_{1}$ denotes the number of steps needed for first failure to happen and $n_{b}$ denotes the number of steps from first failure till complete failure.

We recall that failure of springs starts to happen when $\Delta l \approx f_{1} / k_{2}$ and all springs would have failed when $\Delta l \approx$ $f_{2} / k_{1}$ (see Fig. 3). This gives us the equation

$$
n_{1}^{2}(\Delta x)^{2} \approx\left(\frac{f_{1}}{k_{2}}+l\right)^{2}-l^{2},
$$



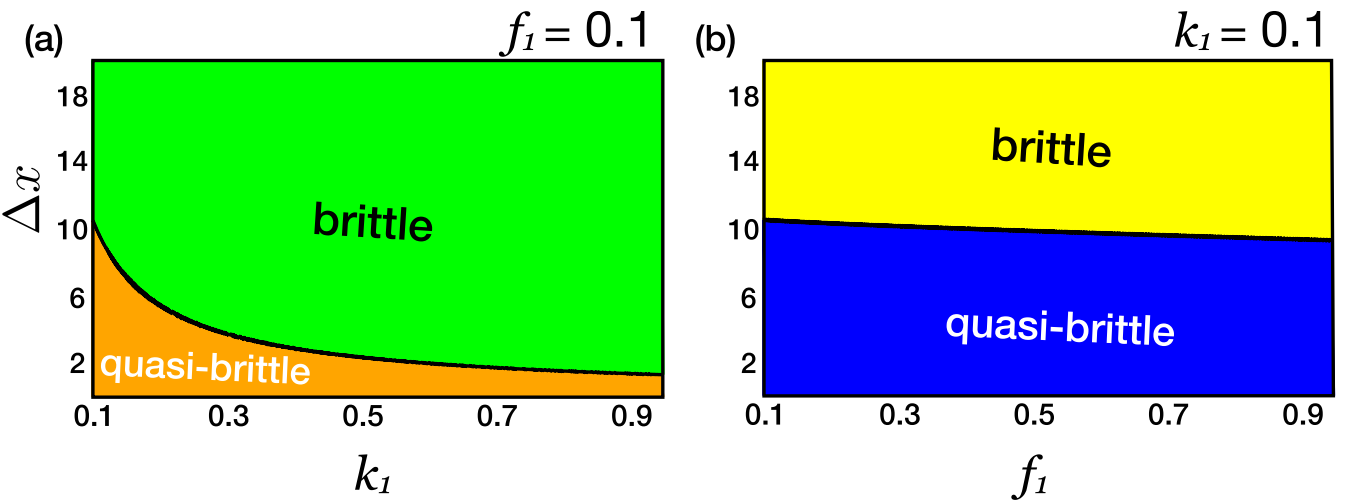

FIG. 7. Phase diagram of the spring system with uniformly distributed threshold strength $(f)$ and spring constant $(k)$, showing a quasibrittle to brittle transition. (a) Phase diagram in the $\Delta x-k_{1}$ plane for fixed $f_{1}=0.1$, (b) in $\Delta x$ - $f_{1}$ plane for fixed $k_{1}=0.1$.

which gives

$$
n_{1} \approx \frac{\sqrt{\left(\frac{f_{1}}{k_{2}}+2 l\right) \frac{f_{1}}{k_{2}}}}{\Delta x}
$$

and similarly, we have

$$
n_{f}^{2}(\Delta x)^{2} \approx\left(\frac{f_{2}}{k_{1}}+l\right)^{2}-l^{2} \Rightarrow n_{f} \approx \frac{\sqrt{\left(\frac{f_{2}}{k_{1}}+2 l\right) \frac{f_{2}}{k_{1}}}}{\Delta x} .
$$

We also define $n_{b}(\Delta x)=n_{f}(\Delta x)-n_{1}(\Delta x)$. The response of the system to shear loading is brittle if all springs break in a single step while the response is quasibrittle in the case when the breaking process occurs over multiple steps. In other words, using notation of Eq. (18), the response to shear loading is brittle if $n_{b}<1$ and the response is quasibrittle if $n_{b} \geqslant 1$. In Fig. 7, we plot the phase diagram of the system in various cases: in Fig. 7(a), the phase diagram is in the $k_{1}-\Delta x$ plane while keeping $f_{1}$ fixed and in Fig. 7(b), the phase diagram is in the $f_{1}-\Delta x$ plane while keeping $k_{1}$ fixed. We note that in each case, a quasibrittle to brittle transition is evident: for sufficiently low values of $\Delta x$, the response is quasibrittle while for high values of $\Delta x$, the response is brittle. Now, in case each $\Delta x$ load is given in fixed $\Delta t$ time, then the load rate $y=\Delta x / \Delta t$ can determine the material response: if $y$ is high, then the response is brittle as $\Delta x$ is high as well, similarly, if $y$ is low, then $\Delta x$ is low which results in a quasibrittle response. This phenomena has been observed to happen in snow [20] and has been studied in Ref. [27] using an FBM model that is similar to our model but less general, in that all the springs in the system have a fixed spring constant $k$. In general, this type of quasibrittle to brittle transition can be considered as a signature of the presence of heterogeneities in the system.

\section{Correlated elasticity and rupture strength}

We now study the spring system for which the values of the spring constant and the rupture strength of the constituent springs are correlated. For simplicity, we assume that the values of the spring constant and the rupture strength are derived from the same interval $[a, b]$, though we note that our results will hold more generally. We consider first a system with positively correlated $k$ and $f$ values, with springs having high rupture strength having relatively higher spring constants as well. Fixing $\epsilon>0$, we define the conditional probability distribution of spring constant conditional on the rupture strength $p_{k \mid f}=U\left[\xi_{f}, \kappa_{f}\right]$, where $U$ denotes the uniform distribution on the interval $\left[\xi_{f}, \kappa_{f}\right]$ with $\xi_{f}=\max (a, f-\epsilon)$ and $\kappa_{f}=\min (f+\epsilon, b)$. Then, with $p_{0}$ denoting the probability distribution of the rupture strength and taken as $U\left[f_{1}, f_{2}\right]$, we have the joint distribution as

$$
p(k, f)=p_{k \mid f}(k) p_{0}(f)=\frac{1}{\left(f_{2}-f_{1}\right)\left(\kappa_{k}-\xi_{k}\right)} .
$$

Unlike the previous case, where the spring constants and the rupture strengths of the springs in the system were taken to be independent with the distribution spread fully on the rectangle $\left[k_{1}, k_{2}\right] \times\left[f_{1}, f_{2}\right]$, in this case, the probability distribution is supported on a strip of width $\sim 2 \epsilon$ around the diagonal $(x, x), a \leqslant x \leqslant b$ of the box $[a, b] \times[a, b]$. We observe a markedly brittle response to displacement controlled shear loading, as shown in Fig. 8, with bundle failure occurring very sharply similar to the case where the spring constants show very little variability while contrasting acutely with the case where the spring constants are spread widely, as in Fig. 4(a).

However, the situation is different when we consider a negative correlation between the $k$ and $f$ values. We again fix an interval $[a, b]$ and $\epsilon>0$. However, we now take the distribution $p_{k \mid f}=U\left[\rho_{f}, \mu_{f}\right]$, with $\rho_{f}=\max (a, a+b-$ $f-\epsilon)$ and $\mu_{f}=\min (a+b-f+\epsilon, b)$. We then have the joint distribution $p(k, f)=p_{k \mid f}(k) p_{0}(f)$, with $p_{0}=U[a, b]$. In this case, the values of $k$ and $f$ are distributed in a region of width $\sim 2 \epsilon$ around the diagonal $(x, a+b-x), a \leqslant x \leqslant b$. In Fig. 8(b), we observe that the system displays a markedly quasibrittle response, comparable to the independent case [Fig. 4(a)]. In other words, the quasibrittle response to loading of the system with independent $k$ and $f$ values can be effectively mimicked by systems with $k$ and $f$ appropriately negatively correlated, even though the support of the values of the spring constants and the rupture strengths of the springs are spread over a much narrower area. To understand this, we note that as the shear displacement is increased, the region $[a, b] \times[a, b]$ in the $k$ - $f$ plane is swept in the counterclockwise direction by lines of the form $f=k \Delta l$, with the area under the line determining the fraction of springs broken (see Fig. 3). In case the values of $k$ and $f$ are negatively correlated, the entire area of $[a, b] \times[a, b]$ has to be swept to cover 

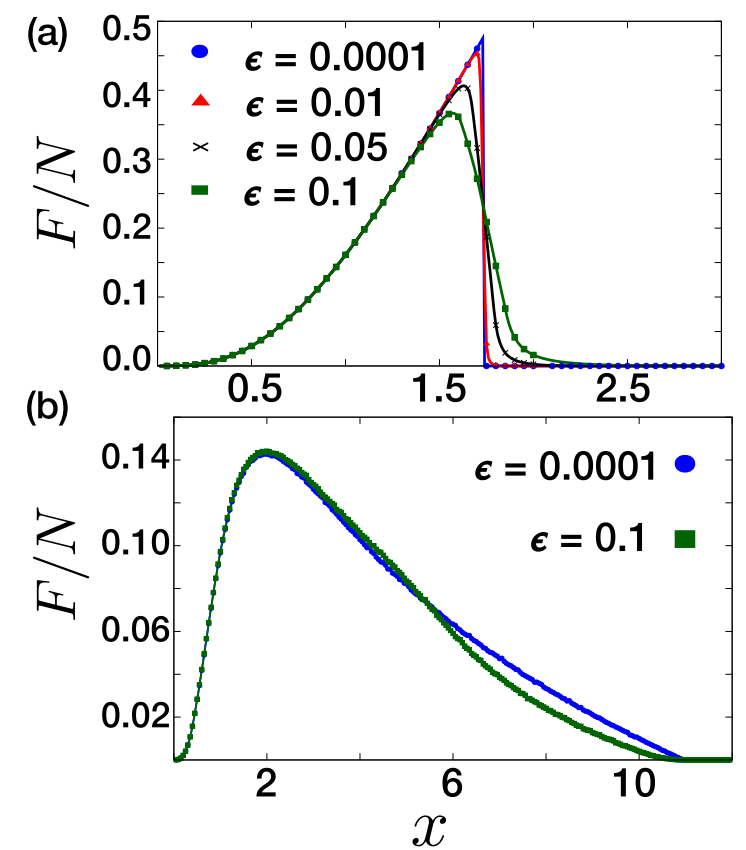

FIG. 8. Force extension curve for systems with (a) positively correlated and (b) negatively correlated $k$ and $f$ values, $\epsilon$ as labeled in inset, distribution of $f$ taken as $U[0.1,1]$ in both cases, computed using Eq. (15) (solid lines) and simulations (points).

all the springs. In the positively correlated case, the $k$ and $f$ values are distributed around the diagonal $(x, x)$, so much lesser area has to swept to cover all springs in the system, hence it exhibits a decidedly brittle response. However, while in the positive correlation case, the maximum load is greater than the independent case, in case of negative correlation, the maximum load is in fact lower than the independent case. Hence, our analysis suggests that, in general, for ensuring a sufficiently high load capacity and also high resistance to failure, it can be important that the distributions of $k$ and $f$ of the springs in the system are independent and are supported over a wide region, essentially resulting in a multi-composite structure. For the particular case of the bacterial cell wall, it is therefore plausible that hydrolytic action effects independent distributions of the elastic properties of the peptide crosslinkers, with wide variability in the stiffness and limited variability in rupture strengths, since this has the effect of making the structure both more resistant to failure under to loading and enhances the load carrying capacity.

\section{NONUNIFORM DISTRIBUTIONS}

So far, we have primarily considered the case with spring constants and rupture strengths of the springs in the system having uniform distribution over appropriate intervals. We now study systems for which the $k$ and $f$ values are independent and follow other distributions. First, we consider the case where the spring constants and the rupture strengths follow Gaussian distributions $N\left(\mu_{0}, \sigma_{0}\right)$ and $N\left(\mu_{1}, \sigma_{1}\right)$, respectively. For a random variable following the $N(\mu, \sigma)$ distribution with mean $\mu$ and standard deviation $\sigma$, it is easy to see that $\approx 99.7 \%$ of values lie within a distance of $3 \sigma$ of the mean $\mu$. We there-
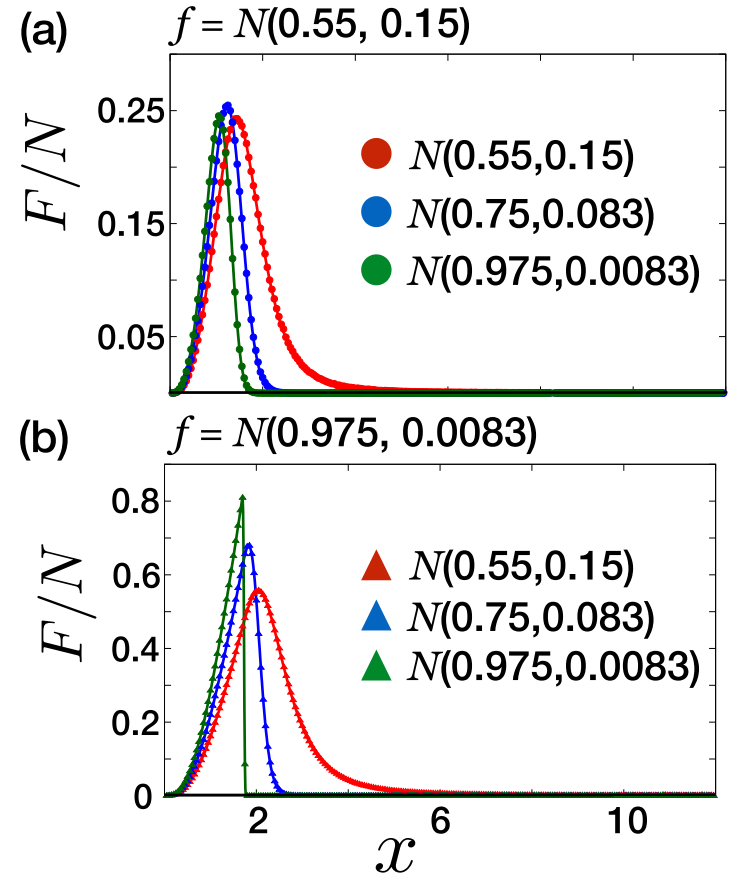

FIG. 9. Force extension curve for systems with $k$ and $f$ values following independent Gaussian distributions. The $f$ values follow (a) $N(0.55,0.15)$ and (b) $N(0.975,0.0083)$ distributions while the distributions of $k$ values are labeled in inset. We compare the curves derived using Eq. (15) (solid lines) and simulations (points). Note that in both cases, system with $k$ distribution $N(0.975,0.0083)$ collapses first and system with $k$ distribution $N(0.55,0.15)$ collapses last.

fore infer that the spring constants and rupture strengths of the constituent springs in the system are essentially distributed in the region $\left[k_{1}, k_{2}\right] \times\left[f_{1}, f_{2}\right]$ with $k_{1}=\mu_{0}-3 \sigma_{0}, k_{2}=\mu_{0}+$ $3 \sigma_{0}, f_{1}=\mu_{1}-3 \sigma_{1}, f_{2}=\mu_{1}+3 \sigma_{1}$. Given $k_{1}, k_{2}, f_{1}, f_{2}$, we solve and get $\mu_{0}=\left(k_{1}+k_{2}\right) / 2, \sigma_{0}=\left(k_{2}-k_{1}\right) / 6, \mu_{1}=$ $\left(f_{1}+f_{2}\right) / 2, \sigma_{1}=\left(f_{2}-f_{1}\right) / 6$. So, we have

$$
p(k, f)=\frac{1}{2 \pi \sigma_{0} \sigma_{1}} e^{-\left[\frac{\left(k-\mu_{0}\right)^{2}}{2 \sigma_{0}^{2}}+\frac{\left(f-\mu_{1}\right)^{2}}{2 \sigma_{1}^{2}}\right]} .
$$

The force extension relation is then computed using Eq. (15) (noting that the integral in this case cannot in general be exactly solved, unlike the uniform case and is therefore numerically approximated) and it is compared with computer simulations of the system as described in Sec. II C, in Fig. 9. Simulations show excellent agreement with theoretical computations, which demonstrates that our theoretical framework is applicable for spring systems irrespective of the distributions followed by $k$ and $f$ values.

In this case, we note that (1) the maximum load is always higher in the Gaussian case as compared to the uniform case and (2) the system in this case collapses at a faster rate, with a significant number of the springs having ruptured at shear displacements much lower than $x_{f}$. This is because in the Gaussian case the $k$ and $f$ values are strongly concentrated around the mean but in the uniform case the values are spread more evenly. To further highlight this, we also consider systems for which the spring constants follow a left truncated and a right truncated normal distributions, essentially supported in 


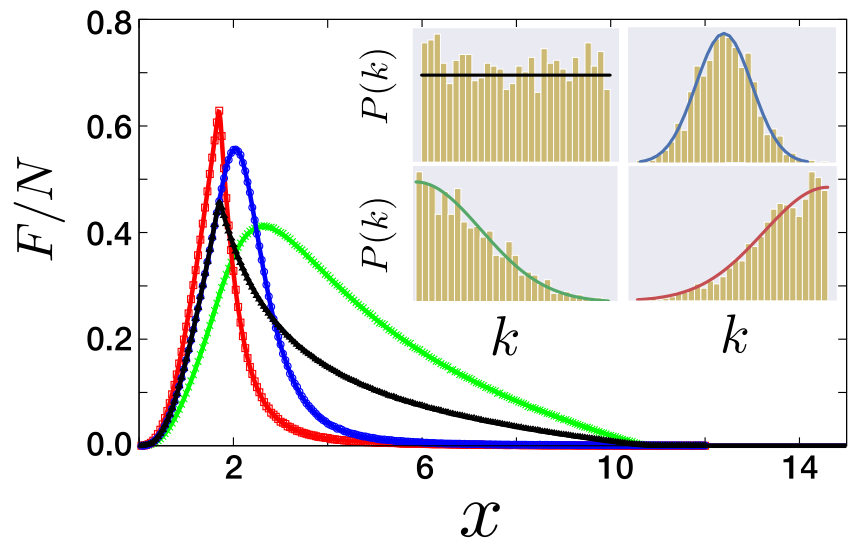

FIG. 10. Force extension curves for systems with $k$ and $f$ values taken independent, with distribution of $f$ fixed as $U[0.95,1]$, computed using Eq. (15) (solid lines) and simulations (points). The $k$ values follow the uniform (black and triangles), Gaussian (blue and circles), left truncated Gaussian (green and lines), right truncated Gaussian (red and squares) distributions, supported on $[0.1,1]$. The distributions are depicted in the inset.

$\left[k_{1}, k_{2}\right]$, with probability distributions given by

$$
p(k)=\delta_{\left[k_{1}, \infty\right)} \frac{\sqrt{2}}{\sigma \sqrt{\pi}} e^{-\frac{\left(k-k_{1}\right)^{2}}{2 \sigma^{2}}}
$$

and

$$
p(k)=\delta_{\left[-\infty, k_{2}\right)} \frac{\sqrt{2}}{\sigma \sqrt{\pi}} e^{-\frac{\left(k-k_{2}\right)^{2}}{2 \sigma^{2}}}
$$

respectively [see Fig. 10 (inset)]. Here, $\sigma=\left(k_{2}-k_{1}\right) / 3$ and the function $\delta_{[a, b]}(x)$ takes value 1 for $a \leqslant x \leqslant b$ and 0 otherwise. In both the uniform and Gaussian cases, wider variability in the $k$ values and limited variability in the $f$ values results in an ideal scenario with a high load carrying capacity and resistance to failure, so we fix this case with $f$ values showing relatively much less variability and following the uniform distribution on a small interval. In Fig. 10, we compare the force extension curves with the spring constants having uniform, Gaussian, left truncated Gaussian and right truncated Gaussian distributions. We observe that the maximum load is highest for the right truncated case and is lowest in the left truncated case, implying that the load capacity is higher when the spring constants are concentrated around a higher value. However, while the systems finally fail at same shear displacement $x_{f}$ for all distributions, the right truncated system fails at the fastest rate and the left truncated system displays a force extension curve that is the slowest to fall, exhibiting substantial quasibrittle response to loading.

We note here that the main results and conclusions of this study are not dependent on the form of the distributions of the values of $k$ and $f$, however, the precise shape of the force extension curve will depend on the distributions. In general, the distributions and the range of the $k$ and $f$ values in the peptidoglycan mesh will be influenced by the rate of incorporation of cell wall material (synthesis rate) and the rate at which hydrolases act to facilitate material removal in the cell wall, factors which modulate the growth rate of the cell as well $[28,29]$. For instance, uniform distribution of the values of $k$ and $f$ will likely occur under conditions resulting in steady incorporation and removal of cell wall material. On the other hand, a concentration of the $k$ and $f$ values closer to the upper limit of the distribution is probably indicative of a higher rate of synthesis as compared to the rate of hydrolysis while a faster rate of hydrolysis might result in a distribution of values concentrated near the lower limit. Since dissimilar growth conditions will likely result in elastic properties of the peptide crosslinkers being differently distributed, our analysis thus suggests that the cell wall adopts disparate constitutive behavior under varying growth conditions. A detailed theoretical study of this will be done in a subsequent work. Another interesting direction in this regard is the possibility of carrying out a form of biological "forensics," to ascertain by carefully analyzing constitutive behavior of isolated cell wall fragments, the conditions undergone by the cell itself during its growth.

\section{DISCUSSION AND CONCLUSION}

In this work, inspired by the crosslinked structure of the bacterial cell wall and to understand the effect of variability in the mechanical properties of the peptide crosslinkers on the structure of the cell wall, we studied the response of a spring system, consisting of several springs joining two adjacent rigid surfaces, to a displacement controlled shear loading. Variability in the mechanical properties of the crosslinkers has been indicated by experimental results on sonication of isolated E.coli sacculi [12] and can arise from action of hydrolases on the cell wall, resulting in a distribution of newly formed and degraded crosslinks. To incorporate variability into the model, the spring constants and rupture strengths of the springs were taken from an appropriate probability distribution. Laying the condition that the distribution of spring constants and rupture strengths are independent, we computed the force extension curve and observed that higher variability in values of the spring constants resulted in a quasibrittle response to loading with a higher value of the shear displacement at which the system collapses while lower variability resulted in a much more brittle response, highlighting the standard problem in engineering of ensuring high orders of stiffness and toughness in a material [1]. On the other hand, while the load capacity increased with lower variability in values of rupture strength, the failure displacement remained independent of the lower limit of its distribution. Thus our work reflected a viable way for providing a high load bearing capacity and resistance to failure to the system is by ensuring a composite structure with wide variability in value of spring constants and low variability in rupture strengths. Since the bacterial cell wall is key to bearing turgor pressure in the cell while being remodelled, with cleaving of crosslinks and insertion of newer glycan strands happening continually, maintaining structural integrity under such conditions is critical to the survival of the cell. Our work suggests that the action of hydrolases, if resulting in good variability in the elasticity of crosslinkers while showing little effect on their rupture strength, can ensure that the structure remains robust enough to sustain high turgor pressure as well as resist mechanical failure. 
We also explored a stepwise loading regime which allowed us to obtain a quasibrittle to brittle transition as the load increases. This transition is a signature of the presence of heterogeneities in the system and hence, is of much importance for experimental detection of the same.

In this work, we have modelled the peptide crosslinkers in the peptidoglycan sacculus as linear springs. Although peptide crosslinkers have often been modelled as linear springs in previous work [26,29,30], nonlinearity in their force extension relation is plausible. Indeed, simulation work has suggested that their force extension curves are well approximated as a worm like chain (WLC) [31]. We note that our theoretical framework can be suitably modified to incorporate nonlinearity in the force extension relation of the peptide crosslinkers, nevertheless, the main results of our study will not change qualitatively.

Several theoretical approaches to modeling the cell wall have taken a continuum theory approach [32-34], which however may not take into account the molecular level architecture and the inhomogeneities inherent in the structure of the cell wall. The peptidoglycan sacculus has a significantly complex structure and we acknowledge that in our simplified model, we have not considered and studied the full range of its design features and their role in ensuring the stability of the cell wall and survival of the cell. Nonetheless, our approach presents a possible first step towards including the inhomogeneities present in the peptidoglycan mesh and its molecular scale architecture in modeling of the cell wall structure and dynamics. In particular, our work anticipates a potentially paradigmatic shift in the coarse grained modeling of the peptidoglycan sacculus by considering variability in the elastic properties of the constituents, leading to a better understanding of the bulk material properties of the sacculus: previous coarse grained models of peptidoglycan have typically assumed uniformity in the elastic properties of glycan strands and peptide crosslinkers [26,29,30]. Future work will involve incorporating the dynamics of the cell wall and the elasticity of the glycan strands into our model alongwith local transfer of load when a crosslink ruptures, from which stress concentrations and pore size distributions can be computed and compared with experiments and simulations, which can lead to further insights on the structure of the cell wall and shed light on its surprising viability against all odds.

\section{ACKNOWLEDGMENTS}

The authors thank B.V. Rao and Himalay Senapati for useful suggestions. I.P. is supported by a DST Inspire Faculty Award (DST/INSPIRE/04/2016/001185).

\section{APPENDIX: SHEAR DISPLACEMENT AND MAXIMUM LOAD CAPACITY}

Here we analyze the shear displacement $x_{m}$ at which the force extension curve attains its maximum as a function of the parameters $f_{1}$ and $k_{1}$, with $f_{2}=k_{2}=1$. Since the shear displacement uniquely determines the spring extension $\Delta l$ by Eq. (4) in the main text, the force extension relation here is taken as a function of $\Delta l$. There are two possibilities- (a) $f_{1} / k_{1} \leqslant f_{2} / k_{2}$ and (b) $f_{2} / k_{2} \leqslant f_{1} / k_{1}$. In case (a), it follows from a straightforward calculation using Eq. (15) in the main text, that the derivative of $F$ with respect to $\Delta l, F^{\prime}(\Delta l)>0$ when $\Delta l<f_{1} / k_{2}$ and that $F^{\prime}(\Delta l)<0$ when $f_{2} / k_{2}=1<$ $\Delta l<f_{2} / k_{1}$, with $F \equiv 0$ when $\Delta l \geqslant f_{2} / k_{1}$. In other words, we have that $F$ strictly increases as a function $\Delta l$ when $\Delta l$ increases between 0 and $f_{1} / k_{2}$ while it strictly decreases when $\Delta l$ increases between $f_{2} / k_{2}=1$ to $f_{2} / k_{1}$ beyond which $F \equiv 0$. Therefore, in this case, the maximum is attained either when $f_{1} / k_{2} \leqslant \Delta l \leqslant f_{1} / k_{1}$ or when $f_{1} / k_{1} \leqslant \Delta l \leqslant f_{2} / k_{2}=1$. With $f_{1} / k_{2} \leqslant \Delta l \leqslant f_{1} / k_{1}$, the force extension relation as in Eq. (15) in the main text, becomes

$$
\begin{aligned}
F= & C(\Delta l)\left[\frac{1}{3(\Delta l)^{2}}\left(k_{2}^{3}(\Delta l)^{2}-f_{1}^{3}\right)\right. \\
& \left.+k_{2}^{2}\left(f_{2}-k_{2} \Delta l\right)-k_{1}^{2}\left(f_{2}-f_{1}\right)\right],
\end{aligned}
$$

where we have

$$
C(\Delta l)=\frac{N \Delta l \sqrt{(l+\Delta l)^{2}-l^{2}}}{2(l+\Delta l)\left(f_{2}-f_{1}\right)\left(k_{2}-k_{1}\right)} .
$$

Now, to compute $x_{m}$, we differentiate $F$ as given by Eq. (A1) with respect to $\Delta l$ and equating with 0 , we get the fifth order polynomial equation, denoted $p_{1}(\Delta l)=0$, given by

$$
\begin{aligned}
& \frac{-4 \Delta l^{5}}{3}+\Delta l^{4}\left(-3-\left(1-f_{1}\right) k_{1}^{2}\right)+\Delta l^{3}\left(\frac{-1}{3}-3\left(1-f_{1}\right) k_{1}^{2}\right) \\
& +\Delta l^{2}\left(3-3\left(1-f_{1}\right) k_{1}^{2}+\frac{f_{1}^{3}}{3}\right)+\Delta l f_{1}^{3}+\frac{f_{1}^{3}}{3}=0 .
\end{aligned}
$$

Next, when $f_{1} / k_{1} \leqslant \Delta l \leqslant f_{2} / k_{2}=1$, we have

$$
\begin{aligned}
F= & C(\Delta l)\left[\frac{\left(k_{2}^{3} \Delta l-k_{1}^{3} \Delta l\right)}{3}+k_{2} \Delta l\left(f_{2}-k_{2} \Delta l\right)\right. \\
& \left.-k_{1}^{2} \Delta l\left(f_{2}-k_{1} \Delta l\right)\right]
\end{aligned}
$$

with $C(\Delta l)$ as in Eq. (A2). Again, we differentiate $F$ with respect to $\Delta l$ and equating with 0 , we get a cubic equation, denoted $p_{2}(\Delta l)=0$, given by

$$
\begin{aligned}
& \Delta l^{3}\left(\frac{4\left(k_{1}^{3}-1\right)}{3}\right)+\Delta l^{2}\left(-3-k_{1}^{2}+4 k_{1}^{3}\right) \\
& \quad+\Delta l\left(\frac{10 k_{1}^{3}-9 k_{1}^{2}-1}{3}\right)+3-3 k_{1}^{2}=0 .
\end{aligned}
$$

Interestingly, the cubic polynomial $p_{2}$ is independent of $f_{1}$ and thus, in case that the maximum is attained when $f_{1} / k_{1} \leqslant$ $\Delta l \leqslant 1$, then the shear displacement at which maxima is attained is independent of $f_{1}$. Since $p_{1}(0)>0$ and $p_{2}(0)>0$ while $p_{1}(1)<0$ and $p_{2}(1)<0$, this implies that both $p_{1}$ and $p_{2}$ have roots in the interval $[0,1]$. It follows from discriminant analysis and by numerical methods that both $p_{1}$ and $p_{2}$ have unique root in this interval. So, the question arises as to when the root of $p_{1}$ lies in the interval $\left[f_{1} / k_{2}, f_{1} / k_{1}\right]$ and likewise, when the root of $p_{2}$ lies in the interval $\left[f_{1} / k_{1}, 1\right]$. We note that

$$
\frac{3 k_{1}^{5}}{f_{1}^{2}\left(k_{1}-1\right)} p_{1}\left(f_{1} / k_{1}\right)=\frac{3 k_{1}^{3}}{\left(k_{1}-1\right)} p_{2}\left(f_{1} / k_{1}\right)=d\left(f_{1}, k_{1}\right),
$$


(a)

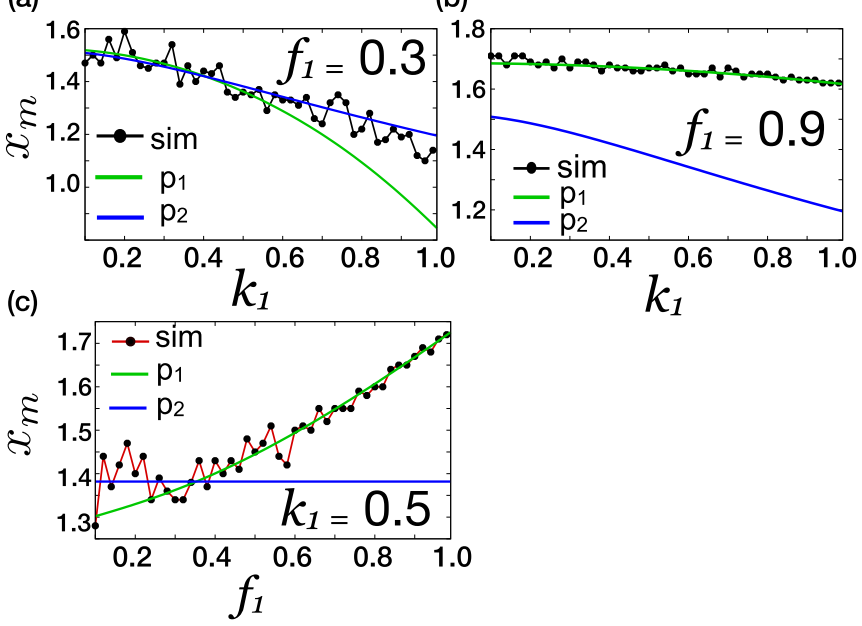

FIG. 11. In (a) and (b), the displacement for which the force extension curve attains maxima, $x_{m}$, is plotted as a function of $k_{1}$, with fixed $f_{1}=0.3$ and fixed $f_{1}=0.9$ respectively. In (c), $x_{m}$ is plotted as a function of $f_{1}$ with fixed $k_{1}=0.5$. The green and blue solid line indicate the value of $x_{m}$ derived from $\Delta l$ taken as the unique solution of polynomials $p_{1}$ and $p_{2}$ while dotted line indicates $x_{m}$ obtained from simulations.

where $d\left(f_{1}, k_{1}\right)$ is the following expression in $f_{1}$ and $k_{1}$ :

$$
\begin{aligned}
& {\left[k_{1}^{4}\left(10 f_{1}-9\right)+k_{1}^{3}\left(12 f_{1}^{2}+f_{1}-9\right)+k_{1}^{2}\left(9 f_{1}^{2}+f_{1}+4 f_{1}^{3}\right)\right.} \\
& \left.\quad+k_{1}\left(9 f_{1}^{2}+4 f_{1}^{3}\right)+4 f_{1}^{3}\right] .
\end{aligned}
$$

Since $p_{1}\left(f_{1} / k_{2}\right)>0, p_{2}(1)<0$ and $k_{1}<1$, it follows that if $d\left(f_{1}, k_{1}\right)>0$ then $p_{1}$ has a root in the interval $\left[f_{1} / k_{2}, f_{1} / k_{1}\right]$ and consequently the maximum of the force extension curve lies in this interval. On the other hand, if $d\left(f_{1}, k_{1}\right)<0$, then $p_{2}$ has a root in the interval $\left[f_{1} / k_{1}, 1\right]$, therefore the force extension curve attains maximum in this interval, in which case, as mentioned, $x_{m}$ is independent of the value of $f_{1}$ since $p_{2}$ is independent of $f_{1}$. We note that when $k_{1} \approx f_{1}$, then $d\left(f_{1}, k_{1}\right)>0$, which implies that in this case, $p_{1}$ has a root between $\left[f_{1} / k_{2}, f_{1} / k_{1}\right]$. Similarly, for high values of $f_{1}$, for instance, with $f_{1} \geqslant 0.8$, we also have $d\left(f_{1}, k_{1}\right)>0$, in which case $x_{m}$ is derived from the root of $p_{1}$ in interval $\left[f_{1} / k_{2}, f_{1} / k_{1}\right]$. But when $f_{1} / k_{1} \ll 1$, then $d\left(f_{1}, k_{1}\right)<0$ and so, $x_{m}$ is derived from the root of $p_{2}$ in the interval $\left[f_{1} / k_{1}, 1\right]$.

Next, in case when $1=f_{2} / k_{2} \leqslant f_{1} / k_{1}$, as before we have $F^{\prime}(\Delta l)>0$ for any $0<\Delta l<f_{1} / k_{2}$ and $F^{\prime}(\Delta l)<0$ when $f_{2} / k_{2}=1<\Delta l<f_{2} / k_{1}$ with $F \equiv 0$ for $\Delta l \geqslant f_{2} / k_{1}$. Therefore the maximum is attained when $f_{1} / k_{2} \leqslant \Delta l \leqslant f_{2} / k_{2}=1$. In this case, $\Delta l$ is also a root of $p_{1}$ as given in Eq. (A3) and is the unique root in interval $\left[f_{1} / k_{2}, 1\right]$. In Fig. 11, we plot $x_{m}$ as a function of $k_{1}$ fixing $f_{1}$ and of $f_{1}$ fixing $k_{1}$. Specifically, in Fig. 11(a) with fixed value of $f_{1}=0.3$, when $k_{1} \leqslant f_{1}$, we have that $1=f_{2} / k_{2} \leqslant f_{1} / k_{1}$ and so, $x_{m}$ is derived from the root of $p_{1}$ (shown in green color). However, when $k_{1} \geqslant f_{1}$, except for a crossover region where $f_{1} \approx k_{1}$ in which the shear displacement at the maximum is derived as a root of $p_{1}$, for larger values of $k_{1}$ with $f_{1} / k_{1} \ll 1$, we see that $x_{m}$ is derived from the root of $p_{2}$ (blue color in Fig. 11). In Fig. 11(b), a high value of $f_{1}=0.9$ is fixed and as discussed, in this case, $x_{m}$ is derived from the root of $p_{1}$ for all values of $k_{1}$. In Fig. 11(c), we have fixed value of $k_{1}=0.5$. We note that for smaller values of $f_{1}$, with $f_{1} / k_{1} \ll 1, x_{m}$ is derived from the root of $p_{2}$ and is, therefore, constant as $f_{1}$ varies. However, as $f_{1}$ approaches $k_{1}$, the behavior crosses over with $x_{m}$ now derived from the root of $p_{1}$, and as $f_{1}$ becomes greater than $k_{1}$, we have $f_{1} / k_{1}>1$ and as discussed, the $x_{m}$ is still derived from the root of $p_{1}$. In all cases, simulation results show excellent agreement with theoretical results.
[1] J. Gordon, The New Science of Strong Materials (Penguin, London, 1991).

[2] A. H. Heuer, D. J. Fink, V. J. Laraia, J. L. Arias, P. D. Calvert, K. Kendall, G. L. Messing, J. Blackwell, P. C. Rieke, D. H. Thompson et al., Science 255, 1098 (1992).

[3] D. M. Popescu and S. X. Sun, J. R. Soc., Interface 15, 20180086 (2018).

[4] B. Ji and H. Gao, J. Mech. Phys. Solids 52, 1963 (2004).

[5] Q. Chen and N. M. Pugno, J. Mech. Behav. Biomed. Mater. 19, 3 (2013).

[6] M. Lenz, M. L. Gardel, and A. R. Dinner, New J. Phys. 14, 033037 (2012).

[7] P. Fratzl, H. S. Gupta, E. P. Paschalis, and P. Roschger, J. Mater. Chem. 14, 2115 (2004).

[8] A. Koch, Bacteria Growth and Form (Springer Berlin, 2001).

[9] W. Vollmer, D. Blanot, and M. A. De Pedro, FEMS Microbiology Rev. 32, 149 (2008).

[10] J. Höltje, Microbiol. Mol. Biol. Rev. 62, 181 (1998).

[11] H. Harz, K. Burgdorf, and J. V Höltje, Anal. Biochem. 190, 120 (1990).

[12] R. W. Verwer, E. H. Beachey, W. Keck, A. M. Stoub, and J. E. Poldermans, J. Bacteriol. 141, 327 (1980).
[13] L. Gan, S. Chen, and G. J. Jensen, Proc. Natl. Acad. Sci. USA 105, 18953 (2008).

[14] M. Schindler, D. Mirelman, and U. Schwarz, Eur. J. Biochem. 71, 131 (1976)

[15] M. A. de Pedro and F. Cava, Front. Microbiol. 6, 449 (2015).

[16] G. Rani and I. Patri, Phys. Rev. E 100, 062408 (2019).

[17] T. K. Lee and K. C. Huang, Curr. Opin. Microbiol. 16, 760 (2013).

[18] H. E. Daniels and H. Jeffreys, Proc. R. Soc. London, Ser. A 183, 405 (1945).

[19] S. Pradhan, A. Hansen, and B. K. Chakrabarti, Rev. Mod. Phys. 82, 499 (2010).

[20] J. Schweizer, Ann. Glaciol. 26, 97 (1998).

[21] N. Ouzounov, J. P. Nguyen, B. P. Bratton, D. Jacobowitz, Z. Gitai, and J. W. Shaevitz, Biophys. J. 111, 1035 (2016).

[22] J. C. Gumbart, M. Beeby, G. J. Jensen, and B. Roux, PLoS Comput. Biol. 10, e1003475 (2014).

[23] M. S. Doulah, Biotechnol. Bioeng. 19, 649 (1977).

[24] Y. Chisti and M. Moo-Young, Enzyme Microb. Technol. 8, 194 (1986).

[25] B. L. Smith, T. E. Schäffer, M. Viani, J. B. Thompson, N. A. Frederick, J. Kindt, A. Belcher, G. D. Stucky, 
D. E. Morse, and P. K. Hansma, Nature (London) 399, 761 (1999).

[26] A. Boulbitch, B. Quinn, and D. Pink, Phys. Rev. Lett. 85, 5246 (2000).

[27] I. Reiweger, J. Schweizer, J. Dual, and H. J. Herrmann, J. Glaciol. 55, 997 (2009).

[28] G. Misra, E. R. Rojas, A. Gopinathan, and K. C. Huang, Biophys. J. 104, 2342 (2013).

[29] A. M. Belgrave and C. W. Wolgemuth, Biophys. J. 104, 2607 (2013).
[30] K. C. Huang, R. Mukhopadhyay, B. Wen, Z. Gitai, and N. S. Wingreen, Proc. Natl. Acad. Sci. USA 105, 19282 (2008).

[31] L. T. Nguyen, J. C. Gumbart, M. Beeby, and G. J. Jensen, Proc. Natl. Acad. Sci. USA 112, E3689 (2015).

[32] H. Jiang and S. X. Sun, Phys. Rev. Lett. 105, 028101 (2010).

[33] Y. Deng, M. Sun, and J. W. Shaevitz, Phys. Rev. Lett. 107, 158101 (2011).

[34] S. Banerjee, N. F. Scherer, and A. R. Dinner, Soft Matter 12, 3442 (2016). 